\title{
Optimization of factor combinations for stem cell differentiations on a design-of-experiments (DOE) microfluidic chip
}

\author{
Lijun $\mathrm{Li}^{\mathrm{a}, \mathrm{b}}$, Deng Tan ${ }^{\mathrm{a}, \mathrm{b}}$, Shuqin Liu ${ }^{\mathrm{a}}$, Ruifeng Jiao ${ }^{\mathrm{a}}$, Xiaofei Yang ${ }^{\mathrm{c}}$, Furong Li ${ }^{\mathrm{c}}$, Hongkai Wu ${ }^{\mathrm{b}, \mathrm{d} *}$, \\ and Wei Huang ${ }^{\mathrm{a}, *}$ \\ a Department of Biology, Southern University of Science and Technology, 1088 Xueyuan Avenue, \\ Nanshan District, Shenzhen, 518055, Guangdong, China. \\ ${ }^{\mathrm{b}}$ Department of Chemistry, The Hong Kong University of Science and Technology, Clear Water Bay, \\ Kowloon, Hong Kong, China. \\ c Translational Medicine Collaborative Innovation Center, The First Affiliated Hospital (Shenzhen \\ People's Hospital), Southern University of Science and Technology, 1017 Dongmen North Road, Luohu \\ District, Shenzhen, 518020, Guangdong, China \\ d Guangzhou First People’s Hospital, 1 Panfu Rd, Yuexiu District,Guangzhou, 510180, Guangdong, \\ China. \\ Corresponding Author: huangw@sustech.edu.cn, chhkwu@ust.hk
}

\section{Supporting material contents}

Figure S1. The photo demonstrating how to set up the DOE chip on a 48-well cell culture plate.

Figure S2. The designings of individual PMMA layers of the 4-input DOE chip.

Figure S3. The illustration of the 7-input DOE chip on a 48-well cell culture plate.

Figure S4. Quantitative measurements of the dispersions of GelRed by the 7-input chip.

Table S1. 4-input DOE designing table.

Table S2. 7-input DOE designing table.

Code S1. The code used to calculate the $p$-values of Friedman's test. 
Figure S1. Photo demonstrating how to mount the device on a 48-well cell culture plate.
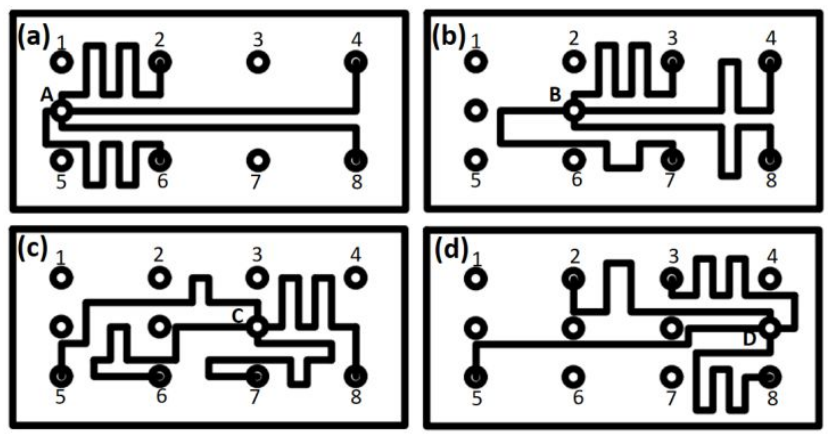

Figure S2. Designing of individual PMMA layers of the 4-input DOE chip.

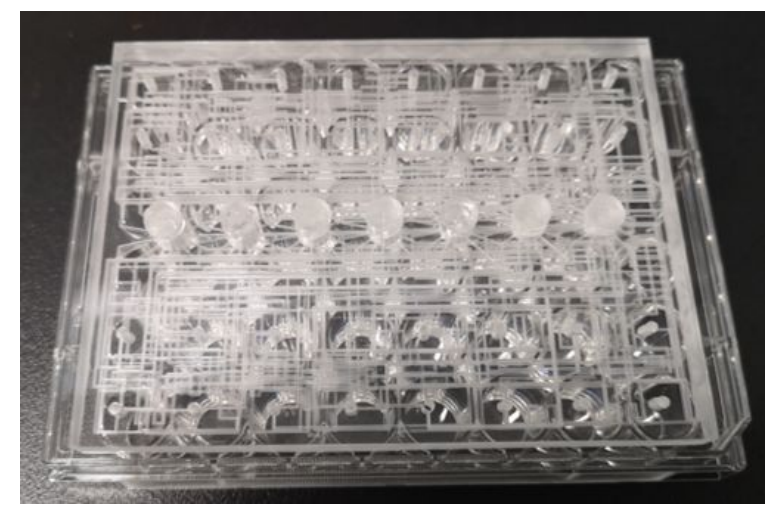

Figure S3. A 7-input and 30-output DOE chip mounting on a 48-well cell culture plate. 


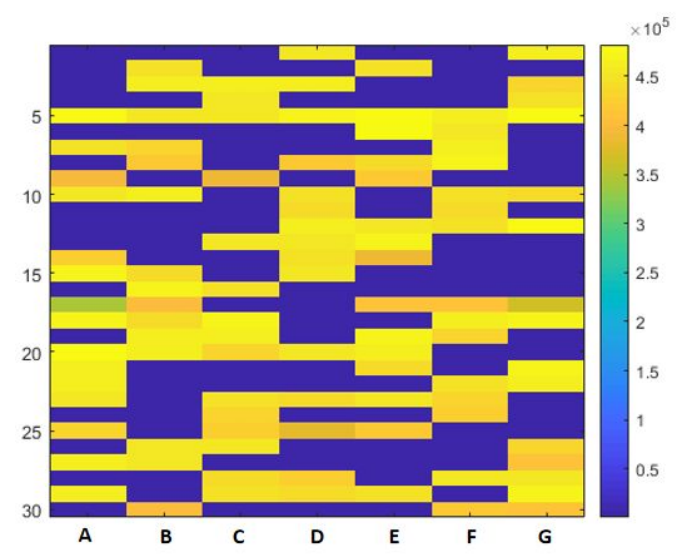

Figure S4. Quantitative measurements of the dispersions of GelRed by the 7-input and 30-ouput chip. The fluorescent signal measurements are presented as a heatmap image. The corresponding values are shown in the color bar on the right.

Table S1. A 4-input DOE designing tale from 'DOE simplified: practical tools for effective experimentation'(Mark J. Anderson and Patrick J. Whitecomb. Third edition. " 1 " represents add and " 0 " represents null.)

\begin{tabular}{|c|c|c|c|c|}
\hline Std & A & B & C & D \\
\hline 1 & 0 & 0 & 0 & 0 \\
\hline 2 & 1 & 0 & 0 & 1 \\
\hline 3 & 0 & 1 & 0 & 1 \\
\hline 4 & 1 & 1 & 0 & 0 \\
\hline 5 & 0 & 0 & 1 & 1 \\
\hline 6 & 1 & 0 & 1 & 0 \\
\hline 7 & 0 & 1 & 1 & 0 \\
\hline 8 & 1 & 1 & 1 & 1 \\
\hline
\end{tabular}


Table S2. A 7-input DOE designing tale from 'DOE simplified: practical tools for effective experimentation" (Mark J. Anderson and Patrick J. Whitecomb. Third edition. " 1 " represents add and " 0 " represents null.)

\begin{tabular}{|c|c|c|c|c|c|c|c|}
\hline Std & A & B & C & D & $E$ & $F$ & $\mathbf{G}$ \\
\hline 1 & 0 & 0 & 0 & 1 & 0 & 0 & 1 \\
\hline 2 & 0 & 1 & 0 & 0 & 1 & 0 & 0 \\
\hline 3 & 0 & 1 & 1 & 1 & 0 & 0 & 1 \\
\hline 4 & 0 & 0 & 1 & 0 & 0 & 0 & 1 \\
\hline 5 & 1 & 1 & 1 & 1 & 1 & 1 & 1 \\
\hline 6 & 0 & 0 & 0 & 0 & 1 & 1 & 0 \\
\hline 7 & 1 & 1 & 0 & 0 & 0 & 1 & 0 \\
\hline 8 & 0 & 1 & 0 & 1 & 1 & 1 & 0 \\
\hline 9 & 1 & 0 & 1 & 0 & 1 & 0 & 0 \\
\hline 10 & 1 & 1 & 0 & 1 & 0 & 1 & 1 \\
\hline 11 & 0 & 0 & 0 & 1 & 0 & 1 & 0 \\
\hline 12 & 0 & 0 & 0 & 1 & 1 & 1 & 1 \\
\hline 13 & 0 & 0 & 1 & 1 & 1 & 0 & 0 \\
\hline 14 & 1 & 0 & 0 & 1 & 1 & 0 & 0 \\
\hline 15 & 1 & 1 & 0 & 1 & 0 & 0 & 0 \\
\hline 16 & 0 & 1 & 1 & 0 & 0 & 0 & 0 \\
\hline 17 & 1 & 1 & 0 & 0 & 1 & 1 & 1 \\
\hline 18 & 1 & 1 & 1 & 0 & 0 & 1 & 1 \\
\hline 19 & 0 & 1 & 1 & 0 & 1 & 1 & 0 \\
\hline 20 & 1 & 1 & 1 & 1 & 1 & 0 & 0 \\
\hline 21 & 1 & 0 & 0 & 0 & 1 & 0 & 1 \\
\hline 22 & 1 & 0 & 0 & 0 & 0 & 1 & 1 \\
\hline 23 & 1 & 0 & 1 & 1 & 1 & 1 & 0 \\
\hline 24 & 0 & 0 & 1 & 0 & 0 & 1 & 0 \\
\hline 25 & 1 & 0 & 1 & 1 & 1 & 0 & 0 \\
\hline 26 & 0 & 1 & 1 & 0 & 0 & 0 & 1 \\
\hline 27 & 1 & 1 & 0 & 0 & 0 & 0 & 1 \\
\hline 28 & 0 & 0 & 1 & 1 & 0 & 1 & 1 \\
\hline 29 & 1 & 0 & 1 & 1 & 1 & 0 & 1 \\
\hline 30 & 0 & 1 & 0 & 0 & 0 & 1 & 1 \\
\hline
\end{tabular}


Code S1. Code used to calculate the $p$-values of Friedman's test

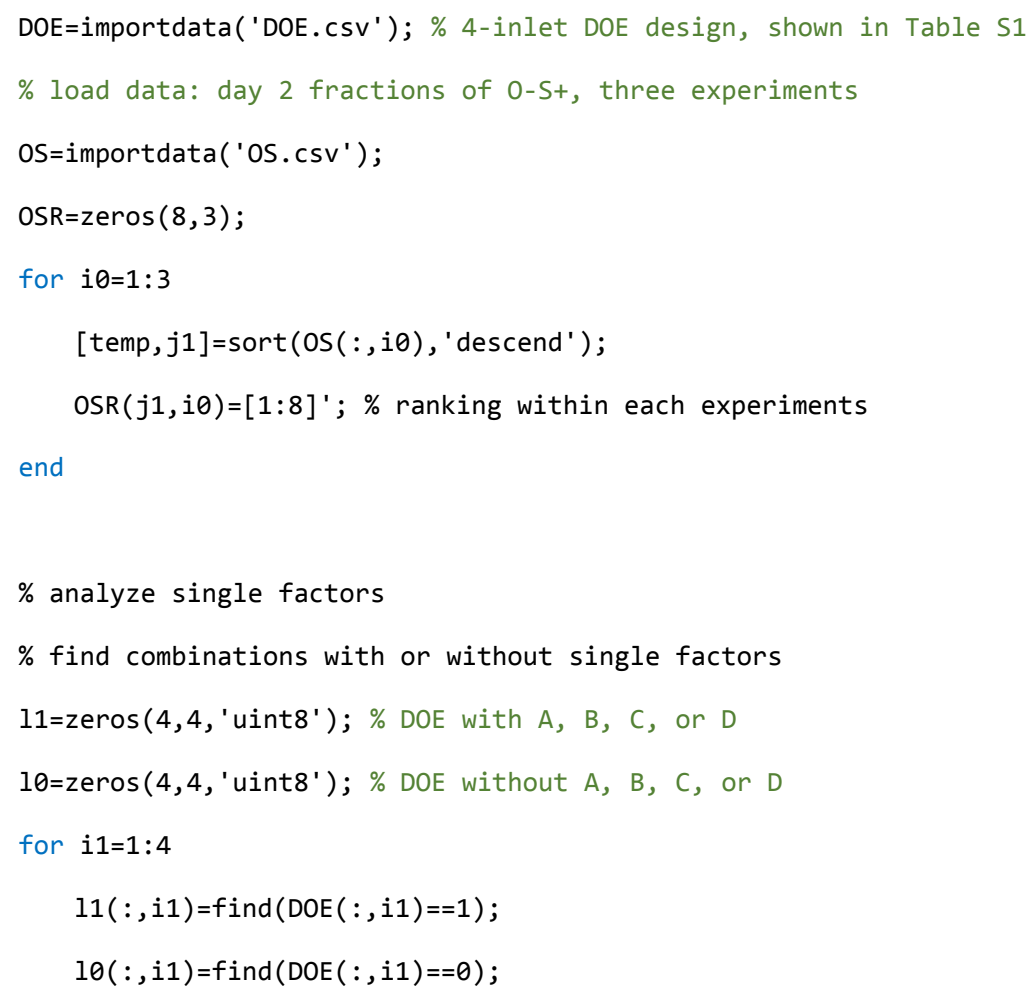




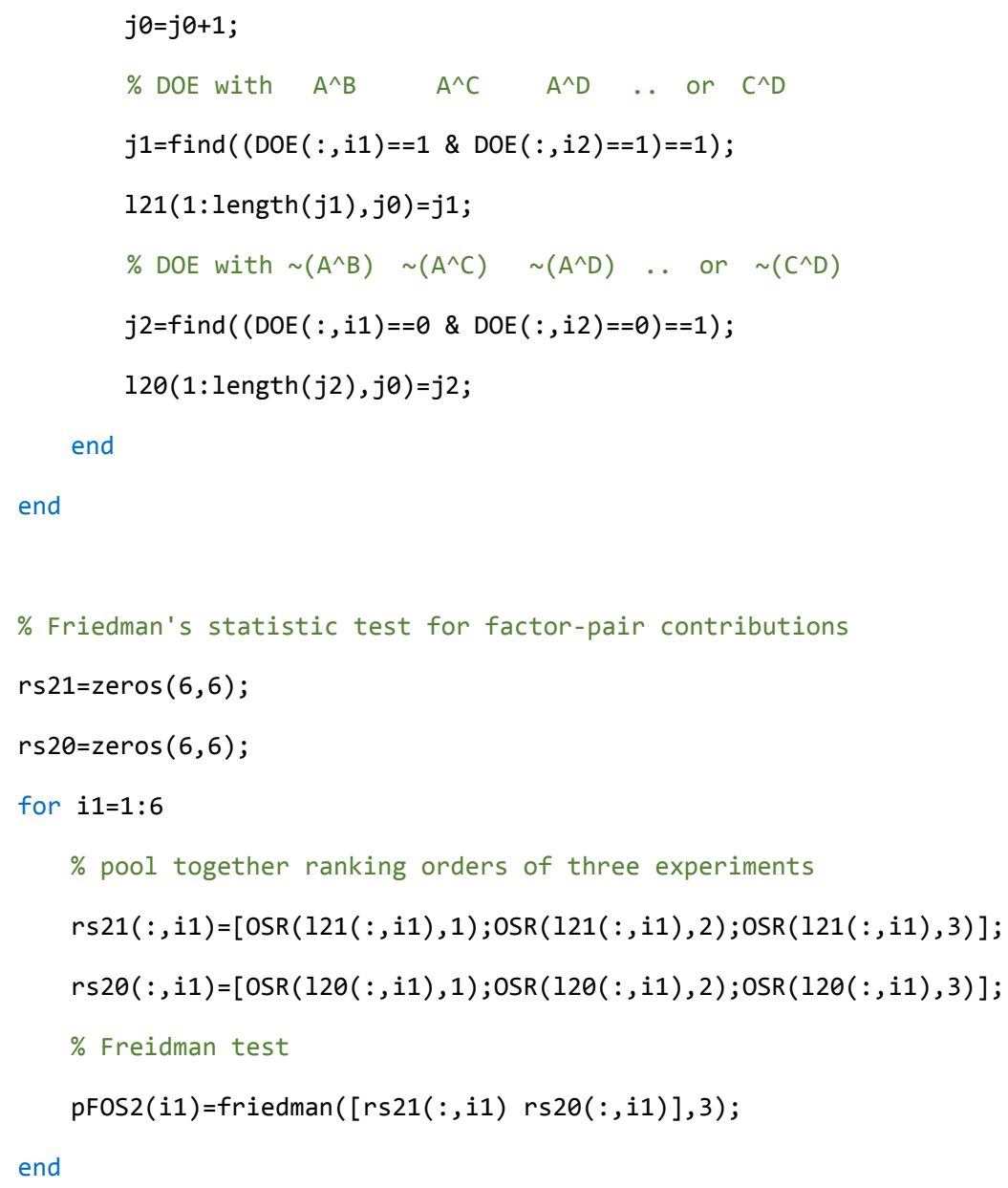

